Available online at GSC Online Press Directory

GSC Advanced Research and Reviews e-ISSN: 2582-4597, CODEN (USA): GARRC2

Journal homepage: https://www.gsconlinepress.com/journals/gscarr

(RESEARCH ARTICLE)

\title{
Ameliorating role of L-carnitine and Ginkgo biloba extract on pentylenetetrazole induced bone marrow injury in epileptic seizures disease in rat
}

\author{
Basiouny Fouad El-sendiony ${ }^{1}$, Mohamed F.F Bayomy ${ }^{2}$ and Ehab Tousson ${ }^{3, *}$ \\ ${ }^{1}$ Biology Department, Preparatory year, Shaqra University, KSA. \\ 2 Zoology Department, Faculty of Science, Menoufiya University, Egypt. \\ ${ }^{3}$ Zoology Department, Faculty of Science, Tanta University, Egypt.
}

Publication history: Received on 25 October 2020; revised on 03 November 2020; accepted on 04 November 2020

Article DOI: https://doi.org/10.30574/gscarr.2020.5.2.0092

\begin{abstract}
Pentylenetetrazole (PTZ) kindling is a model for epilepsy and refers to a phenomenon in which repeated injection of PTZ causes gradual seizure. It was used to identify clinically useful anticonvulsant drugs. The current study aimed to determine the possible protective and ameliorative effects of L-carnitine and Ginkgo biloba leaf extracts (GLE) on bone marrow against PTZ induced epileptic seizures disease in rats. A total of 80 male albino rats were equally divided into eight groups; 1st group was the control; 2nd and 3rd were GLE and L-carnitine groups, respectively; while the 4th group was pentylenetetrazole rat group and the 5th and 6th groups were pre GLE and L-carnitine groups respectively; the 7th and 8th groups were post GLE and L-carnitine, respectively. PTZ leads to decrease hemoglobin (hb), red blood cells (RBCs), hematocrite (Hct), white blood cells (WBCs) and platelets. A variety of histopathological lesions was observed in the bone marrow sections in PTZ group, this variety as marked decrease in the activities of hematopoiesis with an increased in fat cells numbers. Faint or mild positive reaction of PCNA-ir were detected in bone marrow of PTZ rat group. Pre- and post-treatment with GLE and L-carnitine were improved the hematological, histological and immunohistochemical alterations in bone marrow that treated with PTZ.
\end{abstract}

Keywords: Pentylenetetrazole; Seizures; Bone marrow; Ginkgo biloba; L-carnitine; PCNA-ir; Histological; Immunohistochemical

\section{Introduction}

The word "epilepsy "is derived from a greek word epilepsia which means to be seized by forces from without (Berendt, 2002). Epilepsy is the neurological disorder that affect human brain and characterized by seizures affecting people of all ages, race or geographical boundaries (Acharya et al., 2013). There are an estimated at least 65 million people worldwide living with epilepsy (Ngugi, 2010). Classification of epileptic seizures into 2 major classes: partial seizures and generalized seizures. Partial seizures begin in one area of the cerebral cortex, while generalized seizures begin in both cerebral hemispheres. Ther are seizures that are difficult to classify into a particular class, considered as unclassified seizures (Commission on classification and terminology of the ILAE, 1981). The begining of a seizures appears to occur when a small group of abnormal neurons undergo prolonged depolarizations associated with the rapid firing of repeated action potentials. Diagnostic seizure occurs when the electrical discharges of a large number of cells become abnormally linked together, creating a storm of electrical activity in the brain, then seizures may spread to involve adjacent areas of the brain. (Johnston, 2007).

\footnotetext{
* Corresponding author: Basiouny Fouad El-sendiony

Biology Department, Preparatory year, Shaqra University, KSA.
} 
Pentylenetetrazole (PTZ) kindling is an acknowledged model for epilepsy and refers to a phenomenon in which repeated injection of a convulsant causes gradual seizure development culminating in generalized tonic-clonic seizures associated with a cognitive deficit (Sarkisian 2001). PTZ is a pharmaceutical agent that exhibit activity as a central nervous system stimulant. It is considered a non-competitive gamma-aminobutyric acid antagonist. PTZ has been used experimentally to study seizure and identify pharmaceuticals that may control seizure susceptibility (Hansen et al., 2009).

In the recent years, a number of herbal products have been demonstrated to have anticonvulsant activity. Among these herbal products are L-carnitine and Ginkgo biloba extract (Salama et al., 2013; Tousson et al., 2014). L-carnitine is a quaternary compound consists from the amino acids lysine and methionine (Steiber et al., 2004). In eukaryotic cells, it is required for the transport of fatty acids from the intermembraneous space in the mitochondria, into the mitochondrial matrix during the breakdown of lipids (fats) for the generation of metabolic energy (Mehta and Sweety, 2013). Recent studies suggest that L-carnitine may play an important role in oxidative/antioxidative balance and has an antiperoxidative effect on several tissues (Tousson et al., 2014). Two main pharmacologically active groups compounds were present in Ginkgo biloba are the flavonoids and the terpenoids. The flavonoid act mainly as antioxidants (Moreno et al., 2004). GLE reduction tissue levels of reactive oxygen species, inhibits membrane lipid peroxidation (Smith and Luo, 2004). Therefore, the present paper aimed to study the protection and ameliorating role of Ginkgo biloba leaf extract (GLE) and L-carnitine in the hematological, histopathological and immunohistochemical altrations in pentylenetetrazole induced epilepsy in rats.

\section{Material and methods}

\subsection{Animals}

The experiments were performed using 80 male albino rats (Rattus norvigicus) weighing $120 \pm 10 \mathrm{~g}$ and of 7-8 week's age. The rats were kept in the laboratory for one week before the experimental work and maintained on a standard diet (20\% casein, 15\% corn oil, 55\% corn starch, 5\% salt mixture and 5\% vitaminzed starch; Egyptian Company of Oils and Soap Kafr-Elzayat Egypt) and water was available ad libitum. The temperature in the animal room was maintained at $23 \pm 2^{\circ} \mathrm{C}$ with a relative humidity of $55 \pm 5 \%$. Light was on a $12: 12 \mathrm{hr}$ light - dark cycle. The experimental protocol was approved by Local Ethics Committee and Animals Research.

\subsection{Animal grouping}

The rats were equally divided into eight groups (10 rats each). $1^{\text {st }}$ group was control group in which rats never received any treatment. $2^{\text {nd }}$ group was positive control rats (-ve control + L-carnitine ( $300 \mathrm{mg} / \mathrm{kg}$ body weight) once per day every other day for 9 days according to Tousson et al. (2014). while $3^{\text {rd }}$ group was positive control rats (-ve control + GLE (100 mg/kg body weight)) once per day every other day for 9 days according to Rodriguez de Turco et al. (1993). $4^{\text {th }}$ group was the experimental group that treated with pentylenetetrazol (PTZ; $40 \mathrm{mg} / / \mathrm{kg}$ body weight) once per day every other day for 9 days according to Akula et al. (2007); Dhir et al. (2007) and Waggas and Al-Hasani (2010). 5th group The rats were treated with PTZ ( $40 \mathrm{mg} / \mathrm{kg}$ ) once every $48 \mathrm{~h}$ for 9 days after L-carnitine (300 mg/kg) treated once every $48 \mathrm{~h}$ for 9 days. $6^{\text {th }}$ group The rats were treated with PTZ $(40 \mathrm{mg} / \mathrm{kg}$ ) once every $48 \mathrm{~h}$ for 9 days after Ginkgo biloba (100 $\mathrm{mg} / \mathrm{kg}$ ) leaf extract treated once every $48 \mathrm{~h}$ for 9 days. $7^{\text {th }}$ group The rats were injected with PTZ (40 mg/kg) once every $48 \mathrm{~h}$ for 9 days then treated with L-carnitine $(300 \mathrm{mg} / \mathrm{kg})$ once every $48 \mathrm{~h}$ for 9 days. and $8^{\text {th }}$ group The rats were injected with PTZ (40 mg/kg) once every $48 \mathrm{~h}$ for 9 days then treated with Ginkgo biloba (100 mg/kg) leaf extract once every $48 \mathrm{~h}$ for 9 days.

Sample Preparation: Blood samples from each rat were collected from inferior vena cava of each rat in heparinized then analysis of Complete blood picture (CBC) parameters and estimation of Hemoglobine (hb), Red blood cells (RBCs), Hematocrite (Hct), White blood cells (WBCs) and Platelets by automatic method (sysmex kx- 21n automated hematology analyzer; JAPAN CARE CO., LTD).

\subsection{Histopathological investigation}

Immediately after decapitation, animals were dissected, bone marrow from animals of different groups were quickly removed, washed in $0.9 \%$ saline solutions and samples of bone marrow were fixed in $10 \%$ neutral buffered formalin. After fixation, specimens were dehydrated in an ascending series of alcohol, cleared in two changes of xylene and embedded in molten paraffin (mp. $50-58^{\circ} \mathrm{C}$ ). Sections of 7 microns thickness were cut using rotary microtome and mounted on clean slides. Sections were stained with Ehrlich's haematoxylin and counterstained with eosin as a routine method by Bancroft and Stevens (1990). 


\subsection{Immunohistochemical detection of (PCNA-ir)}

Expression of proliferating cell nuclear antigen immunoreactivities (PCNA-ir) was detected using avidin Biotin

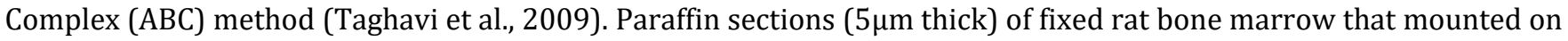
gelatin chromalum-coated glass slides were dewaxed and rehydrated sections were washed in distilled water for 5 min, rinsed in PBST for $10 \mathrm{~min}$ and incubated with 10\% normal goat serum for 15 min to reduce non-specific background staining. Then, the sections were incubated with anti-rabbit PCNA for 1-2 hours at room temperature. The sections after 5 times baths in PBST were incubated with biotinylated goat antirabbit immuoglobulin (Nichirei, Tokyo, Japan). The sections after 5 times baths in PBST were further incubated with Avidin Biotin Complex (ABC: Nichirei, Tokyo, Japan) for 1 hour at RT. The criterion for a positive reaction confirming the presence of PCNA is cells with brown nuclear staining. For the negative control, the primary antibody was omitted to guard against any false positive results which might develop from a non-specific reaction. Brightness, contrast were adjusted using Adobe Photoshop software. Image analysis was adjusted using PAX-it image analysis software.

\subsection{Statistical Analysis}

Data were expressed as mean values \pm SEM and statistical analyses were performed using SPSS statistical version 16 software package (SPSS $®$ Inc., USA). The criterion for statistical significance was set at $\mathrm{p}<0.01$.

\section{Results}

Figures (1-5) show that both blood Hb, RBCs, Hct, WBCs and platlets levels in Pentylenetetrazole group (G4) showed significant decrease when compared with control (G1), L-carnitine (G2) and GLE (G3) groups. In contrast, both blood Hb, RBCs, Hct, WBCs and platlets in pretreated PTZ group (G4) with L-carnitine and GLE groups (G5 \& G6) were significantly increased when compared with PTZ group (G4). both blood Hb, RBCs, Hct, WBCs and platlets in post treated PTZ group (G4) with L-carnitine and GLE groups (G7 \& G8) were significantly increased when compared with PTZ group (G4).

H b level (g/dI)

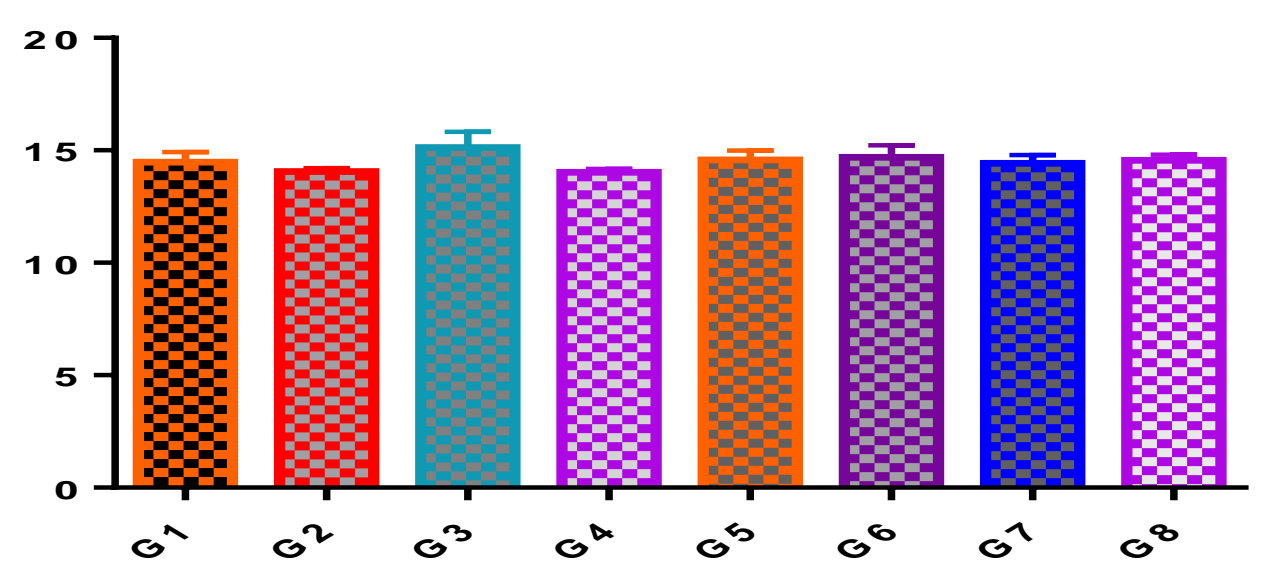

Figure 1 Changes in $\mathrm{Hb}$ level in different groups under study. Where G1, Control group; G2, Ginkgo biloba group; G3, Lcarnitine group; G4, Pentylenetetrazole group; G5, Pre-treated Pentylenetetrazole with Ginkgo biloba group; G6, Pretreated Pentylenetetrazole with L-carnitine group; G7, Post-treated Pentylenetetrazole with Ginkgo biloba group; G8, Post-treated Pentylenetetrazole with L-carnitine group. 


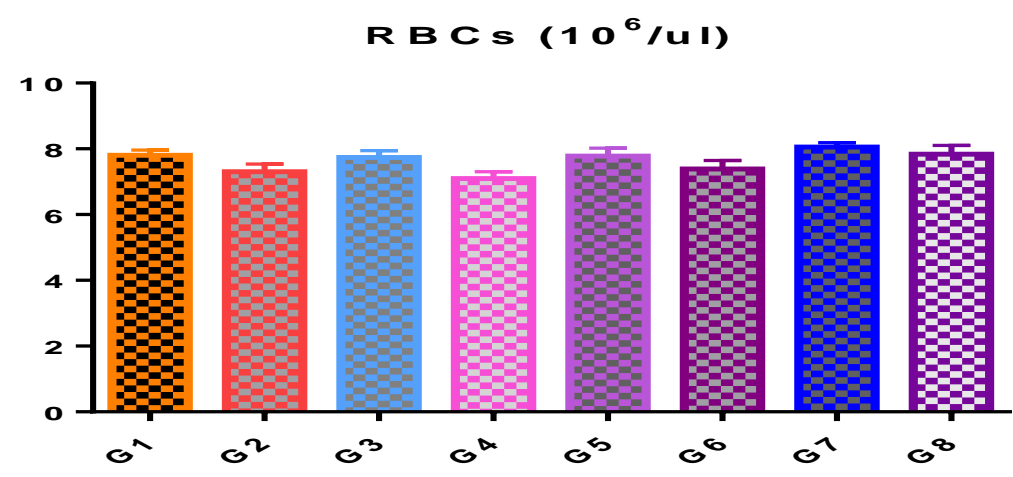

Figure 2 Changes in RBCs count in different groups under study. Where G1, Control group; G2, Ginkgo biloba group; G3, L-carnitine group; G4, Pentylenetetrazole group; G5, Pre-treated Pentylenetetrazole with Ginkgo biloba group; G6, Pretreated Pentylenetetrazole with L-carnitine group; G7, Post-treated Pentylenetetrazole with Ginkgo biloba group; G8, Post-treated Pentylenetetrazole with L-carnitine group.

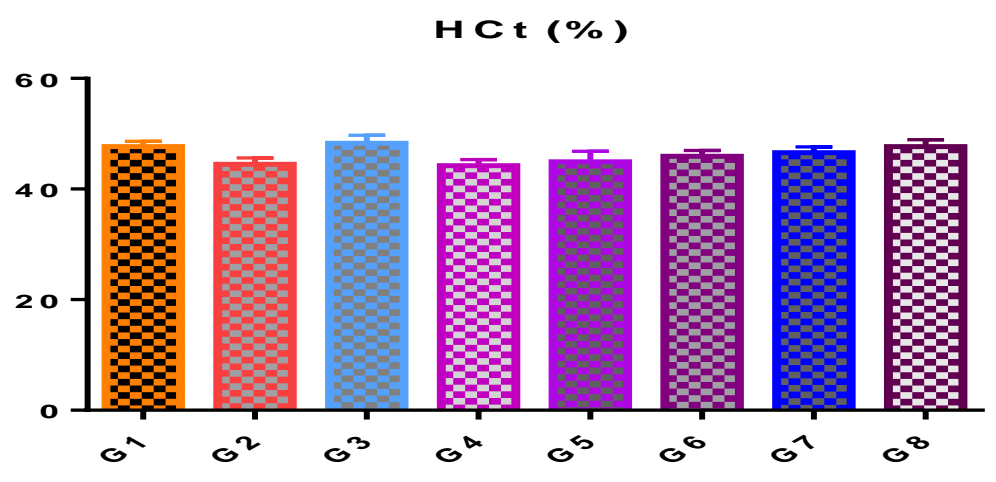

Figure 3 Changes in Hct level in different groups under study. Where G1, Control group; G2, Ginkgo biloba group; G3, L-carnitine group; G4, Pentylenetetrazole group; G5, Pre-treated Pentylenetetrazole with Ginkgo biloba group; G6, Pretreated Pentylenetetrazole with L-carnitine group; G7, Post-treated Pentylenetetrazole with Ginkgo biloba group; G8, Post-treated Pentylenetetrazole with L-carnitine group.

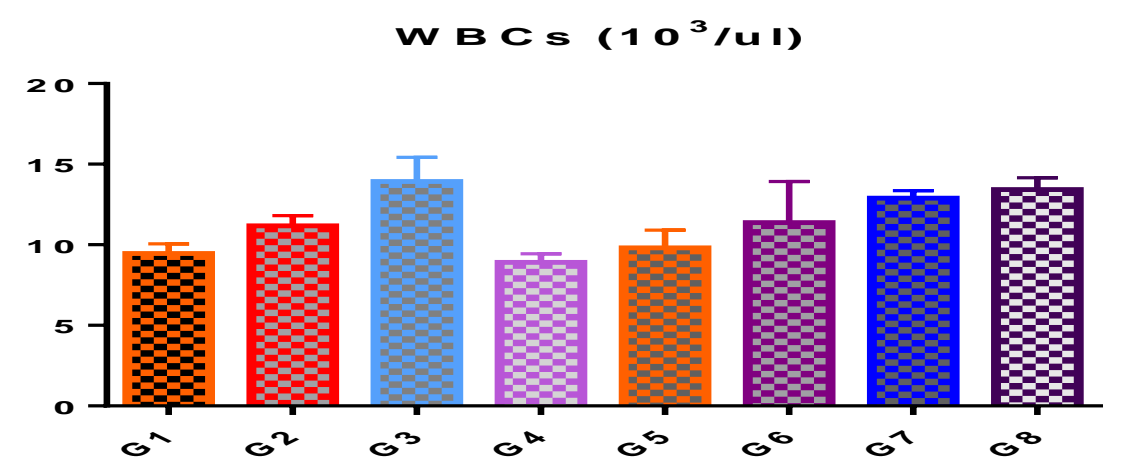

Figure 4 Changes in WBCs count in different groups under study. Where G1, Control group; G2, Ginkgo biloba group; G3, L-carnitine group; G4, Pentylenetetrazole group; G5, Pre-treated Pentylenetetrazole with Ginkgo biloba group; G6, Pre-treated Pentylenetetrazole with L-carnitine group; G7, Post-treated Pentylenetetrazole with Ginkgo biloba group; G8, Post-treated Pentylenetetrazole with L-carnitine group. 


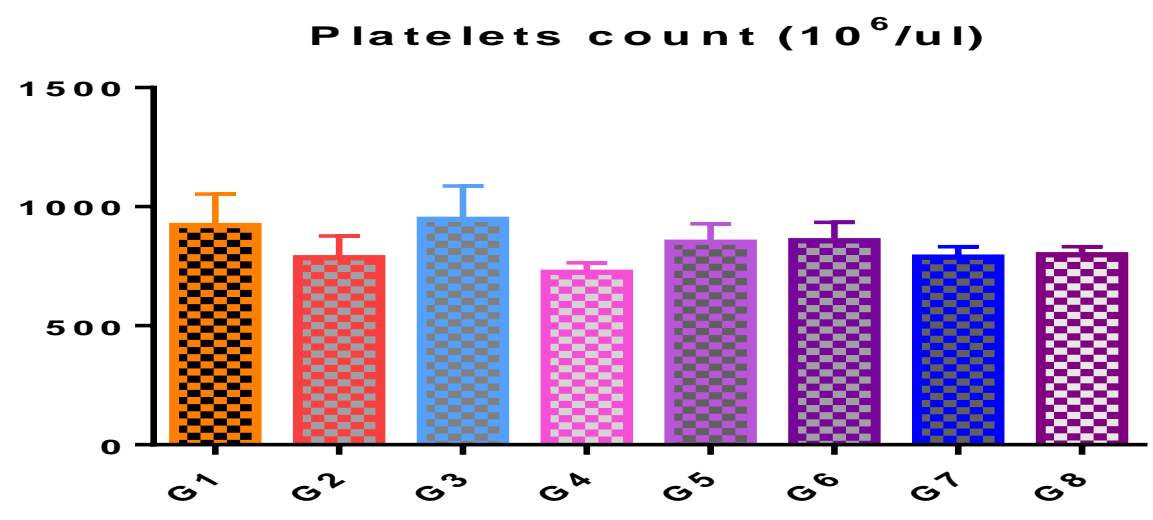

Figure 5 Changes in Platelets count in different groups under study. Where G1, Control group; G2, Ginkgo biloba group; G3, L-carnitine group; G4, Pentylenetetrazole group; G5, Pre-treated Pentylenetetrazole with Ginkgo biloba group; G6, Pre-treated Pentylenetetrazole with L-carnitine group; G7, Post-treated Pentylenetetrazole with Ginkgo biloba group; G8, Post-treated Pentylenetetrazole with L-carnitine group.

\subsection{Histopathological findings in the bone marrow}

Representative coronal sections of $\mathrm{H} \& \mathrm{E}$ stain obtained from the male rat bone marrow in different groups under study are shown at the light microscope levels in Figures $(6 \mathrm{~A}-6 \mathrm{H})$. Coronal sections in bone marrow of control, L-carnitin and GLE groups revealed a normal histopathological structure with an increased the activity of hematopoiesis (Figs. 6A-6C). The histopathological examination of PTZ rat bone marrow revealed various histopathological changes, as marked decrease in the activities of hematopoiesis with an increased in fat cells numbers in addition to atrophy where seen in some granules (Fig. 6D). Mild degree of improvement were observed in bone marrow sections in pro treated PTZ with GLE or with L-carnitin, this improvement as mild increased the activity of hematopoiesis and decrement of the fat cells (Figs. 6E-6F). Moderate degree of improvement were observed in bone marrow sections in post treated PTZ with GLE with marked increased the activity of hematopoiesis (Figs. 6G). Bone marrow sections in post treated PTZ with L-carnitin showed mild increased the activity of hematopoiesis and of a moderate number of fat cells $(6 \mathrm{H})$.

\subsection{Immunohistochemistry findings in the bone marrow}

The detection and distribution of PCNA immunoreactivity (PCNA-ir) are shown on bone marrow sections in the different groups under study (Figures 7A-7H). Strong positive reaction for PCNA-ir were detected in the bone marrow of control (G1), L-carnitine (G2) and GLE (G3) groups (Figs. 7A-7C).

In PTZ rat group (G4), Faint or mild positive reaction for PCNA -ir were detected in the bone marrow (Figs.7D).

Bone marrow sections in pro-treated PTZ with L-carnitine (G5) revealed mild to moderate positive reaction for PCNA -ir (Fig. 7E). In contrast; bone marrow sections in pro-treated PTZ with GLE (G6) revealed faint positive reaction for PCNA-ir (Fig. 7F). The intensity of PCNA-ir on bone marrow sections in pro-treated PTZ with L-carnitine were increased when compared with pro-treated PTZ with GLE. Bone marrow sections in both post-treated PTZ with L-carnitine (G7) and with GLE (G8) revealed moderate reaction for PCNA-ir (Figs 7G- 7H). The intensity of PCNA-ir on bone marrow sections in post-treated PTZ with L-carnitine were increased when compared with pro-treated PTZ with GLE. The intensity of PCNA-ir in the bone marrow sections were depressed in pro- treated PTZ with L-carnitine or GLE when compared with post treated PTZ with L-carnitine or GLE (Figs 7E- $7 \mathrm{H}$ ). 

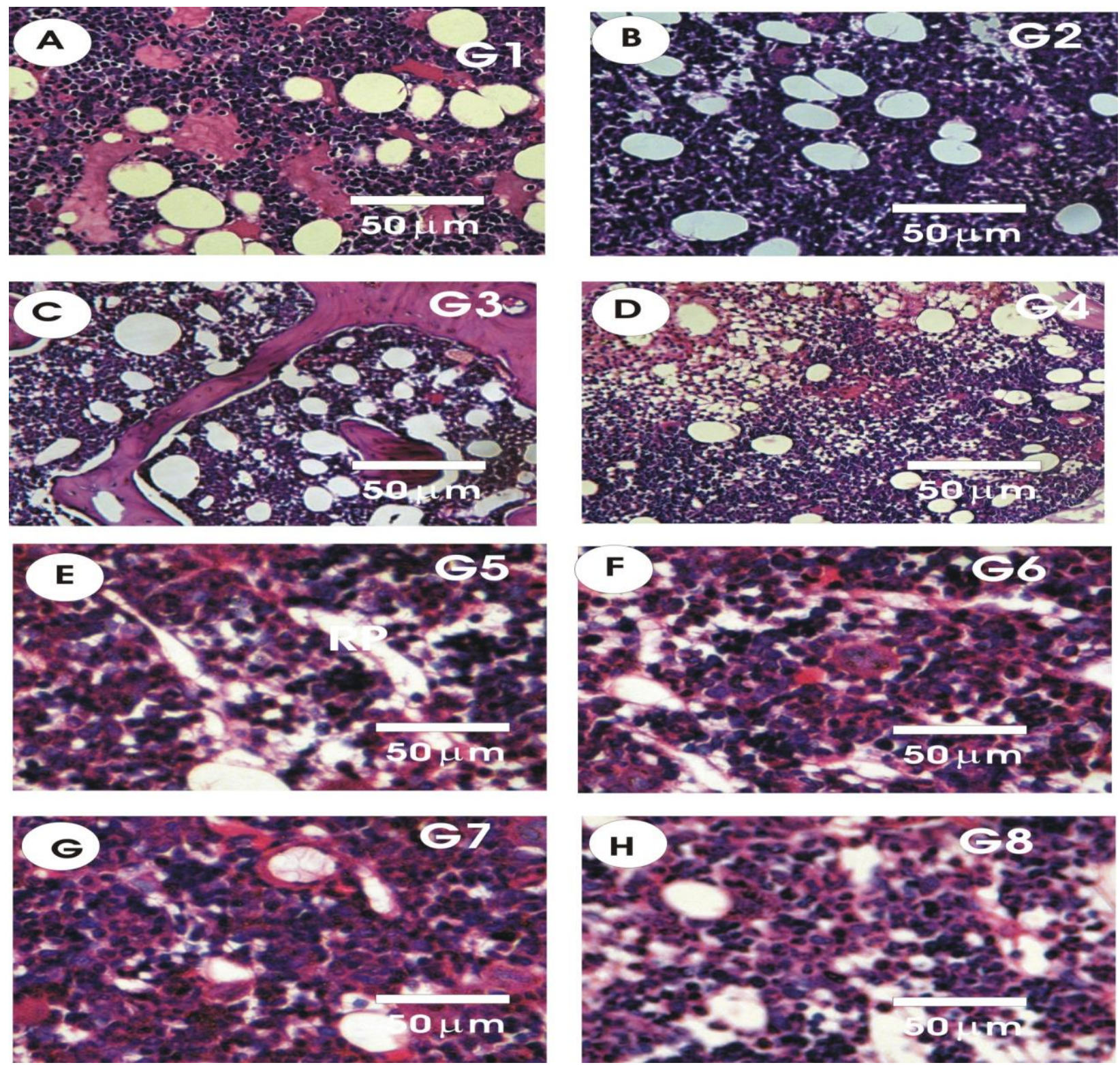

Figures 6A-6H Photomicrographs of rat bone marrow sections in the different experimental groups stained with Haematoxylin \& Eosin. A-C: Rat bone marrow sections in control (G1); L-carnitin (G2) and GLE (G3) groups revealed a normal histopathological structure. D: Rat bone marrow sections of PTZ group (G4) revealed marked decrease in the activities of hematopoiesis with an increased in fat cells numbers in addition to atrophy where seen in some granules. E: bone marrow sections in pro-treated PTZ with L-canitin revealed mild degree of improvement as mild increased the activity of hematopoiesis. F: bone marrow sections in pro-treated PTZ with GLE revealed mild increased the activity of hematopoiesis and decrement of the fat cells. G: bone marrow sections in post treated PTZ with L-canitin, revealed marked increased the activity of hematopoiesis. H: bone marrow sections in post treated PTZ with GLE revealed mild increased the activity of hematopoiesis and of a moderate number of fat cells. 

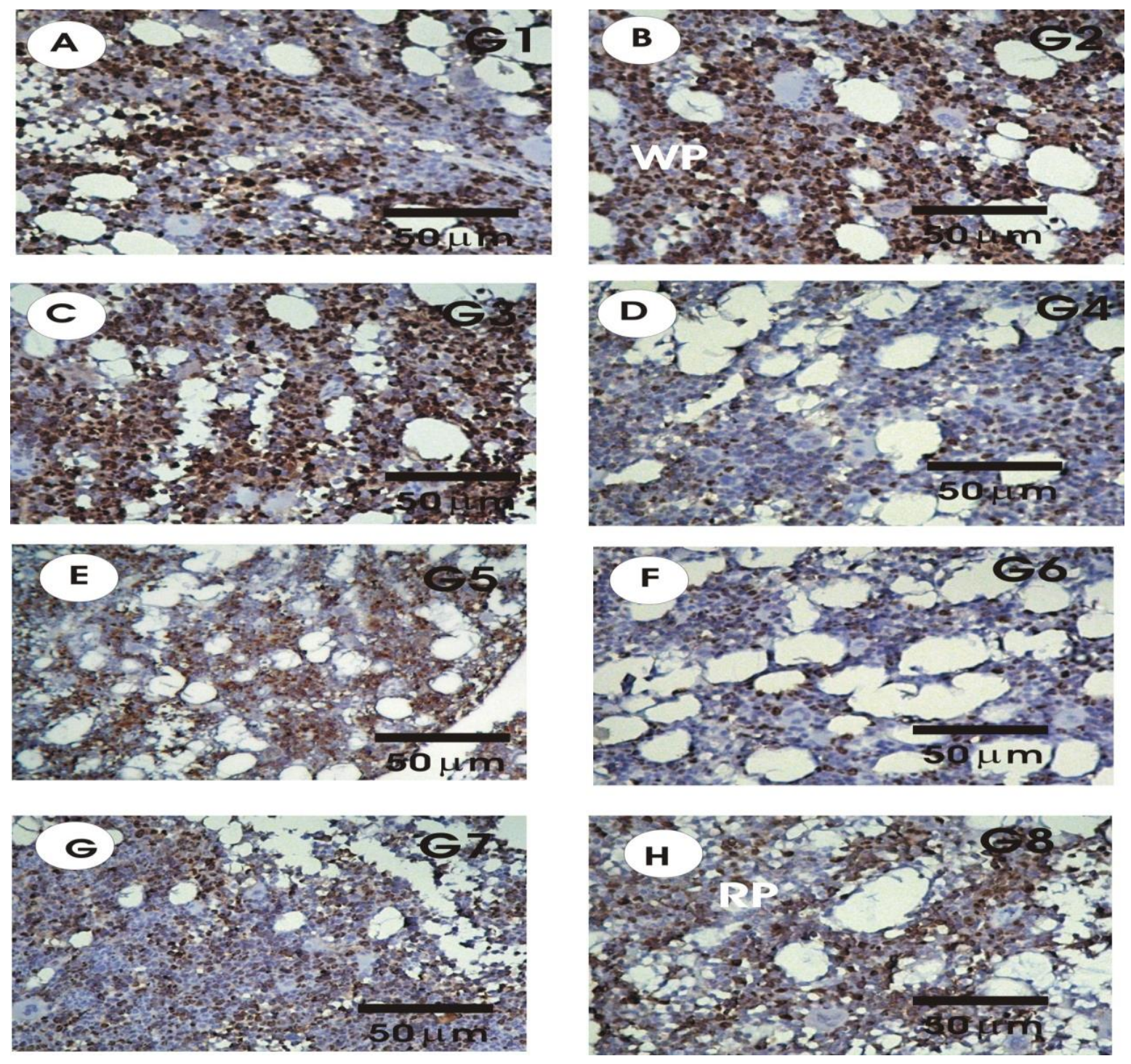

Figures 7A-7H Photomicrographs of PCNA immunoreactivity (PCNA-ir) in the coronal sections of rat bone marrow. AC: Strong positive reaction for PCNA-ir in the control (A), L-carnitine (B) and GLE (C) groups, respectively. D: Faint or mild positive reaction for PCNA -ir were detected in the bone marrow in PTZ rat group (G4). E: Bone marrow sections in pro-treated PTZ with L-carnitine (G5) revealed mild to moderate positive reaction for PCNA -ir. F: Bone marrow sections in pro-treated PTZ with GLE (G6) revealed faint positive reaction for PCNA-ir. . G\&H: Bone marrow sections in both post-treated PTZ with L-carnitine (G7) and with GLE (G8) revealed moderate reaction for PCNA-ir.

\section{Discussion}

Epilepsy is one of the most common neurologic problems all over the world, being associated with paroxysmal discharge of cerebral neurons and is characterized by several symptoms including alterations of behaviors and consciousness (Godlevskii et al., 2004).

Pentylenetetrazole, a GABA receptor antagonist, is a well-established animal model to assess efficacy of drugs against generalized absence seizures in humans (Liu et al. (2012). Pentylenetetrazole-induced seizure activity mimics the increased oxidative stress in the brain by altering membrane phospholipid metabolism and ultimately resulting in the release of free radicals (Corvino et al., 2013).

L- Carnitine is a naturally occurring endogenous compound in all mammalian species and the most widely known function is its role as an important transporter of long chain fatty acids into mitochondria for $\beta$-oxidation (Bieber, 1988). L-carnitine, among a wide array of target neuroprotective agents, is of particular interest in neurons due to its role in 
amino acid synthesis, ATP metabolism, mitochondrial fatty acid transport, as well as its antioxidant effects (Gülc,in 2006; Silva-Adaya et al. 2008; Jones et al. 2010; Wu et al. 2011).

EGb761 is a standardized extract of GB leaves and has antioxidant properties as a free radical scavenger. EGb761 decreases tissue levels of reactive oxygen species, inhibits membrane lipid peroxidation and, with its anti-plateletactivating factor activity, contributes to improvements in cerebral insufficiency (Liu et al., 2006).

This study conducts a hematological, histological and immunohistochemical investigation into whether L-carnitine and Ginkgo biloba have a protective and ameliorative effect on pentylenetetrazole induced tissue damage in bone marrow in male rats. In the present study, PTZ administration in rats over a period of 9 injection resulted in progressive seizure intensity (tonic-clonic), increased mortality rate and alteration in animal behavior.

A significant decrease in the hematological parameters include Hb, RBCs, Hct, WBCs and Platelets after PTZ administration when compared to control animals. Similarly, Daoud et al. (2002) The mean levels of HB, MCV, and MCH were lower in children with first febrile seizure than in children in a control group, although the differences were not significant. This similar other study was found that the proportions of cases with low levels of HB, HCT, MCV, MCH, and ferritin were higher among children with FC than among controls and the differences were statistically significant (Naveed and Billoo 2005). Further, compared the levels of HB, MCV, and serum iron among controls and patients with FC, and they reported that iron deficiency anemia is significantly more frequent among the cases than among the controls (Pisacane et al., 1996). In contrast, a study was suggested that iron deficiency anemia may protect children against febrile seizure (Kobrinsky et al., 1995).

A significant reduction pre and post treatment with L-carnitine and Ginkgo biloba induced improvement in hematological parameters but not significantly. Similarly, Hurot et al. (2002) have reported that L-carnitine administration has increased levels of $\mathrm{Hb}$ in in the groups receiving L-carnitine as compared with the groups not receiving L-carnitine; also they suggested that L-carnitine improves erythropoietin efficiency as compared with control group. Oral administration of L-carnitine has decreased platelet activity (MPV) significantly, this result may be due to decreasing of calcium ion influx into platelets which in turn causes platelet inactivation (Bykov et al., 2003). a significant increase in WBCs (Ozsoy et al., 2011). Carnitine replenishment improved myelotoxicity as manifested by correction of anemia, leucopenia and thrombocytopenia (Hossam et al., 2014).

The administration of oral Gingko biloba showed an improvement in the undesirable effects,this may be a result of the anti oxidative activity of ginkgo biloba that restored the membrane permeability which was altered bythe free radicals (Shafaq and Tabassum, 2010), and /or reduction of platelet - activating factor and improvement of peripheral circulation (Gamal et.al.,2011; Chung et.al., 1999). As compared to cisplatin alone, the combined administration of rabbits with oral Ginkgo-biloba plus intrapretoneal cisplatin resulted in an improvement in the total WBCs count, RBCs count, hemoglobin and finally the platelets (Elawady et al., 2011).

A variety of histopathological lesions was observed in the bone marrow sections in PTZ group (G4), this variety as marked decrease in the activities of hematopoiesis with an increased in fat cells numbers in addition to atrophy where seen in some granules. Mild degree of improvement were observed in bone marrow sections in pro treated PTZ with GLE or with L-carnitin (G5 \& G6), this improvement as mild increased the activity of hematopoiesis and decrement of the fat cells. Moderate degree of improvement were observed in bone marrow sections in post treated PTZ with GLE with marked increased the activity of hematopoiesis (G7). Bone marrow sections in post treated PTZ with L-carnitin showed mild increased the activity of hematopoiesis and of a moderate number of fat cells (G8).

L-carnitine stimulates erythropoiesis, which was expressed by a significant increase of erythroid progenitors in bone marrow and other hematopoietic organs (Kitamura et al., 2005). Carnitine replenishment improved myelotoxicity as manifested by correction of anemia, leucopenia and thrombocytopenia (Hossam et al., 2014).

Bone marrow cells undergoing induced osteogenic or adipogenic differentiations in the presence of EGb 761 show increase and decrease in mineralization and adipogenesis respectively. EGb 761 led to increase in apoptotic cells and ROS, an important upstream signal (Jyoti Gautam et al., 2012).

Faint or mild positive reaction for PCNA -ir were detected in the bone marrow of PTZ rat group (G4). Bone marrow sections in pro-treated PTZ with L-carnitine (G5) revealed mild to moderate positive reaction for PCNA -ir. In contrast; bone marrow sections in pro-treated PTZ with GLE (G6) revealed faint positive reaction for PCNA-ir. Bone marrow sections in both posttreated PTZ with L-carnitine (G7) and with GLE (G8) revealed moderate reaction for PCNA-ir. 
L-carnitine showed a cytoprotective effect on leucocytes against Cisplatin (CDDP) induced toxicity in rat. Apoptotic effect of CDDP was found in lymph node in high degree and thymus in low degree. Carnitine supplementation to diet decreased apoptotic effect of cisplatin. CD68 positive cells were found similar in control and L-carnitine groups. While CD68 positivity was found in low degree in CDDP group, it was moderate in L-Car + CDDP group (Ertan et al., 2012).

The anti-apoptotic properties of EGb reported in the studies (Lucinda et al. 2013 and Smith and Luo 2004) could result from the antioxidant action of the flavonoids present in the extract, which attenuate the reactive oxygen species by chelating pro-oxidant transitional metal ions. Likewise, Brayboy et al. (2001) reported that EGb was effective in protecting an osteoblast-like cell, from death when exposed to free radicals, thus increasing their proliferation in vitro. This similar with other study showed that EGb was effective in improving the expression of Bcl-2 in the femoral trabecular bone suggesting that one of the mechanisms involved in the bone increase is to reduce the osteoblast death (Leda et al., 2014).

\section{Conclusion}

It can be concluded that pre and post-treatment pentylenetetrazole with Ginkgo biloba and L-carnitine prevented oxidative damage by inhibiting ROS production. This study suggests that the ability of Ginkgo biloba and L-carnitine to induced improvement in hematological parameters and increased the activity of hematopoiesis.

\section{Compliance with ethical standards}

\section{Acknowledgments}

To all participants in the study. To Prof. Ehab Tousson for his supported in all the time of research. To Prof. Mohammed F Bayomy continuous encouragement throughout this work.

Disclosure of conflict of interest

There is no conflict of interest.

Statement of ethical approval

Animals used for research only within an ethical framework.

\section{References}

[1] Acharya U. R., Sree S. V., Swapna G., Martis R. J. and Suri J. S. Automated EEG analysis of epilepsy: A review. Knowledge-Based Systems, 2013; 45, 147-165.

[2] Akula K. K., Dhir A. and Kulkarni S. K. Pro-convulsant effect of cefazolin sodium against pentylenetetrazol or Picrotoxin- induced convulsions in mice. Indian J. Exp. Biol., 2007; 45:720-725.

[3] Bancroft J. D. and Stevens G. A. Theory and Practice of Histological Techniques; 2nd Ed. Churchill Livingstone, London. 1990.

[4] Berendt M., Gredal H., Pedersen L. G., Alban L. and Alving J. A Cross-Sectional Study of Epilepsy in Danish Labrador Retrievers: Prevalence and Selected Risk Factors. Journal of Veterinary Internal Medicine 2002;16:262-268.

[5] Bieber L. L. Carnitine. Annu Rev Biochem. 1988; 57:261-283.

[6] Brayboy J. R., Chen X.W. and Lee Y. S. Anderson. The protective effects of Ginkgo biloba extract (EGb 761) against free radical damage osteoblast-like bone cells (MC3T3-E1) and the proliferative effects of EGb 761 on these cells. Nutr. Res, 2001; 21:1275-1285.

[7] Bykov I., Jarvelainen H. and Lindros K. L-carnitine alleviates alcohol-induced liver damage in rats: role of tumour necrosis factor -alpha. Alcohol and alcoholism 2003; Vol.38:No.5:pp. 400-406.

[8] Chung S., Harris A., KrisTinsson K., Kagemann C. and Ritch R. Ginko bilboa extract increases ocular blood flow velocity. Journal of Ocular Pharmacology and Therapeutics.1999; 15:233-240. 
[9] Commission on Classification and Terminology of the International League Against Epilepsy. Proposal for revised clinical and electrographic classification of epileptic seizures. Epilepsia 1981; 22:489-501.

[10] Corvino V., Marchese E., Michetti F. and Geloso M. C. Neuroprotective strategies in hippocampal neurodegeneration induced by the neurotoxicant trimethyltin. Neurochem Res. 2013; 38 (2):240- 53.

[11] Daoud A. S., Batieha A., Abu-Ekteish F., Gharaibeh N., Ajlouni S. and Hijazi S. Iron status: a possible risk factor for the first febrile seizure. Epilepsia. 2002; 43 (7):740-3.

[12] Dhir A., Naidu P. S. and Kulkarni S. K. Neuroprotective effect of nimesulide, a preferential COX-2 inhibitor, against pentylenetetrazol (PTZ)-induced chemical kindling and associated biochemical parameters in mice.Seizure., 2007; 16(8):691-7.

[13] Elawady A. Ibrahim., Medhat W. Ismail and Hossam S. Kareem Alleviation of Cisplatin-induced Toxicities by Encapsulation IntoLiposomes in the Absence or Presence of GinkgoBiloba. Journal of American Science, 2011; $7(10)$.

[14] Ertan ORUC, Adem KARA, Ismail CAN., Ali KARADENIZ.and Nejdet Simsek Caspase-3 and CD68 Immunoreactivity in Lymphoid Tissues and Haematology of Rats Exposed to Cisplatin and L-carnitine. Kafkas Univ Vet Fak Derg. 2012; 18 (5):871-878.

[15] Gamal E., Aly E., Sherief S., Talaat S. and Sallam M. FTIR Assessment of the effect of Ginko Biloba Leave extract (EGb761) on mammalian retina. Cell Biochem.Biophys. 2011; 61 (1):169 -177.

[16] Godlevskii L. S., Stepanenko K. I., Lobasyuk B. A., Sarakhan E. V. and Bobkova L. M. The effects of electrical stimulation of the paleocerebellar cortex on penicillininduced convulsive activity in rats. Neurosci Behav Physiol. 2004; 34:797-802.

[17] Gü lc. in, Antioxidant and antiradical activities of l-carnitine, Life Sci. 2006; 78:803- 811.

[18] Hansen S. L., Nielsen A. H., Knudsen K. E., Artmann A., Petersen G., Kristiansen U., Hansen S. H. And Hansen H. S. Ketogenic diet is antiepileptogenic in pentylenetetrazole kindled mice and decrease levels of $\mathrm{N}$ acylethanolamines in hippocampus. Neurochem Int 2009; 54:199-204

[19] Hossam M. Arafa., Raed S. Ismail., Nesreen Nabil and Adel M. Mostafa Carnitine Deficiency: A Causative Clue or a Sequel in Carboplatin Myelosuppression. Journal of Cancer Research Updates. 2014; 3:226-235.

[20] Hurot J., Cucherat M., Haugh M. and Fouque D. Effects of L-Carnitine Supplementation in Maintenance Hemodialysis Patients: A Systematic Review. J Am Soc Nephrol 2002; 13:708-714.

[21] Johnston M. V. Seizures in childhood. In: Kleigman RM, Behrman RE,Jenson HB, Stanton BP. Nelson Text Book of Pediatrics 18th Edition Philadelphia: Saunders Elsevier. 2007; p.2457-8.

[22] Jones L. L., McDonald D. A. and Borum P. R. Acylcarnitines: role in brain. Prog Lipid Res. 2010; 49:61-75.

[23] Jyoti Gautam., Priyanka Kushwaha., Gaurav Swarnkar., Vikram Khedgikar., Geet K. Nagar., Divya Singh., Vishal Singh., Manish Jain., Manoj Barthwal and Ritu Trivedi EGb 761 promotes osteoblastogenesis, lowers bone marrow adipogenesis and atherosclerotic plaque formation. International journal of phytotherapy and phytopharmacology. 2012; 19(12).

[24] Kitamura Y., Satoh K., Satoh T., Takita M. and Matsuura A. Effect of L-carnitine on erythroid colony formation in mouse bone marrow cells. Nephrol Dial Transplant. 2005; 20:981-984.

[25] Kobrinsky N. L., Yager J. Y., Cheang M. S., Yatscoff R. W. and Tenenbein M. Does iron deficiency raise the seizure threshold? Journal of Child Neurology. 1995; 10:105-9.

[26] Leda M.F. Lucinda., Beatriz J.V. Aarestrup., Joanna S. Brandão., Vera M. Peters., João E. de P. Reis and Martha de 0. Guerra The effect of Ginkgo biloba extract treatment in the Bcl-2 expression by osteoblasts in the femoral trabecular bone of Wistar rats with glucocorticoid-induced osteoporosis. 2014; 24 (3): 363-366.

[27] Liu M., Sheng Z., Cai L., Zhao K., Tian Y. and Fei J. Neuronal conditional knockout of NRSF decreases vulnerability to seizures induced by pentylenetetrazol in mice. Acta Biochim Biophys Sin (Shanghai) 2012; 44:476-482.

[28] Liu S. Q., Yu J. P., Chen H. L., Luo H. S., Chen S. M. and Yu H. G. "Therapeutic effects and molecular mechanisms of Ginkgo biloba Extract on liver fibrosis in rats," The American Journal of Chinese Medicine, 2006; vol. 34, no. 1:pp. 99-114. 
[29] Lucinda M., Aarestrup B. J., Peters V. M., Reis J. E., Oliveira R. S. and Guerra M. O. The effect of the Ginkgo biloba extract in the expression of Bax, Bcl-2 and bone mineral content of Wistar rats with glucocorticoid-induced osteoporosis Phytother. Res.; 2013: 27. 515-520.

[30] Mehta and Sweety, "Activation and transportation of fatty acids to the mitochondria via the carnitine shuttle". Pharmaxchange.info. Retrieved 2013; 2014-02-01.

[31] Moreno S., Carvalho J. J., Nascimento A. L. R., Freitas R. S., Diré G. F., Lima E. A., Lima-Filho G. L., Rocha E. K. and Bernardo-Filho M. Biodistribution of sodium pertechnetate and light microscopy of organs isolated from the rats: Study of the effects of a Ginkgo biloba extract. Pak. J. Nutr. 2004; 3:64-7.

[32] Naveed-Ur-Rehman and Billo A. G. Association between iron deficiency anemia and febrile seizures. Journal of College of Physicians and Surgeons Pakistan. 2005; 15(6):338-40.

[33] Ngugi A. K., Bottomley C., Kleinschmidt I., Sander J. W. and Newton C. R. Estimation of the burden of active and life-time epilepsy: A meta-analytic approach. Epilepsia. 2010; 51(5):883-890.

[34] Ozsoy S. Y., Ozsoy B., Ozyildiz Z.and Aytekin I. Protective effect of L-carnitine on experimental lead toxicity in rats: a clinical, histopathological and immunohistochemical study. Biotech Histochem. 2011; 86(6):436-43.

[35] Pisacane A., Sansone R., Impagliazzo N., Coppola A., Rolando P. and D'Apuzzo A. Iron deficiency anaemia and febrile convulsions: case-control study in children under 2 years. BMJ. 1996; 313(7053):343.

[36] Rodriguez de Turco E. B., Droy-Lefaix M. T., and Bazan N. G. Decreased electroconvulsive shock-induced diacylglycerols and free fatty acid accumulation in the rat brain by Ginkgo biloba extract (EGb 761): selective effect in hippocampus as compared with cerebral cortex. J. Neurochem. 1993; 61:1438-1444.

[37] Salama A., Kasem S., Tousson E. and Elsisy MK. L-carnitine and vitamin E alleviate reproductive toxicity caused by triton WR 1339 in male albino rats. Toxicology and Industrial Health.2013. DOI: $10.1177 / 0748233712472523$.

[38] Sarkisian M. R. Overview of the Current Animal Models for Human Seizure and Epileptic Disorders.Epilepsy Behav. 2001; 2:201-16.

[39] Shafaq N. and Tabassum M. Antioxidant effect of carmosine pretreatment on CP-induced renal oxidative stress in rats in rats. Indian J. clin.Biochem. 2010; 25(1):86-91.

[40] Silva-Adaya D., Perez-De La Cruz V. and Herrera-Mundo M. N. Excitotoxic damage, disrupted energy metabolism, and oxidative stress in the rat brain: antioxidant and neuroprotective effects of L-carnitine. J Neurochem. 2008; 105:677-89.

[41] Smith and Luo Studies on molecular mechanisms of Ginkgo biloba extract Appl. Microbiol. Biot. 2004; 64. 465472

[42] Taghavi M., Moallem S. and Alavi S. The evaluation of single dose effects of methamphetamine on proliferating and apoptosis of sperm germ cells in mature rat. J Isfahan Med School. 2009; 27(97):100-109.

[43] Tousson E., Hafez E., Zaki S. and Gad A. P53, Bcl-2 and CD68 expression in response to amethopterin-induced lung injury and ameliorating role of l-carnitine: Biomed Pharmacother, 2014; v. 68:p. 631-9.

[44] Waggas A. and Al-Hasani R. Neurophysiological study on possible protective and therapeutic effects of Sidr (Zizyphus spina-christi) leaf extract in male albino rats treated with pentylenetetrazol. Saudi Journal of Biological Sciences, 2010; 17(4): 269- 274.

[45] Wu C., Gopal K., Gross G. W., Lukas T. J. and Moore E. J. An in vitro model for testing drugs to treat tinnitus. Eur J Pharmacol 2011; 667:188-194. 